\title{
Microwave and RF Photonic Applications of Integrated Kerr Micro-Combs
}

\author{
Xingyuan Xu, ${ }^{1}$ Jiayang Wu, ${ }^{1}$ Sai T. Chu, ${ }^{3}$ Brent E. Little, ${ }^{4}$ Roberto Morandotti, ${ }^{5}$ Arnan Mitchell, ${ }^{2}$ and \\ David J. Moss ${ }^{1, *}$ \\ ${ }^{1}$ Centre for Micro-Photonics, Swinburne University of Technology, Hawthorn, VIC 3122 Australia \\ ${ }^{2}$ School of Engineering, RMIT University, Melbourne, VIC 3000, Australia \\ ${ }^{3}$ Department of Physics and Material Science, City University of Hong Kong, Hong Kong, China. \\ ${ }^{4} X i$ 'an Institute of Optics and Precision Mechanics Precision Mechanics of CAS, Xi'an, China. \\ ${ }^{5}$ INRS -Énergie, Matériaux et Télécommunications, Varennes, Québec, J3X 1S2, Canada. \\ *dmoss@swin.edu.au
}

\begin{abstract}
We report applications of integrated Kerr micro-combs to RF photonic systems and demonstrate a wide range of advanced functions including a microwave photonic intensity differentiator, filters and true time delays. The on-chip Kerr optical comb is CMOS-compatible and serves as a high-performance multi-wavelength source for transversal filter functions, thus greatly reducing the cost, size, and complexity of the system. OCIS codes: (060.5625) Radio frequency photonics; (190.4390) Nonlinear optics, integrated optics; (280.5110) Phased-array radar.
\end{abstract}

Photonic integrated circuits that exploit nonlinear optics for all-optical signal processing have been demonstrated, particularly in silicon, including all-optical logic [1], demultiplexing from 160Gb/s [2] to over 1Tb/s [3], to optical performance monitoring using slow light at speeds of up to 640Gb/s [4-5], all-optical regeneration [6], and many others. The efficiency of all-optical devices depends on the waveguide nonlinear parameter, $\gamma=\omega n_{2}$ / $c A_{\text {eff. }}$ Although silicon can achieve extremely high nonlinearities, it suffers from high nonlinear losses due to twophoton absorption (TPA) and the resulting free carriers. Even if the free carriers are eliminated by p-i-n junctions, silicon's poor nonlinear figure of merit $\left(\mathrm{FOM}=n_{2} /(\beta \lambda)\right.$, where $\beta$ is the TPA) of 0.3 in the telecom band is too low to achieve high performance. While TPA can be turned to advantage for all-optical functions [7-9], for the most part silicon's low FOM in the telecom band is a limitation.

In 2008-2010, new CMOS compatible platforms for nonlinear optics were introduced, including Hydex [10 18] and silicon nitride [19]. These platforms exhibit negligible nonlinear absorption in the telecom band, and have revolutionized micro-resonator optical frequency combs. The first integrated CMOS compatible integrated optical parametric oscillators were reported in 2010 [11, 19], showing that Kerr frequency comb sources could be realised in chip form by using ring resonators with relatively modest Q-factors. Following this, a stable modelocked laser with pulse repetition rates at $200 \mathrm{GHz}$ was reported [12]. The success of this platform arises from its very low linear loss, its moderate nonlinearity $\left(\gamma \cong 233 \mathrm{~W}^{-1} \mathrm{~km}^{-1}\right)$ and negligible TPA [18].

Here, we review radio-frequency (RF) and microwave photonic applications of micro-combs. Optical frequency combs, and micro-combs in particular, are ideal sources for microwave and RF transversal filters [20]. Radar mapping, measurement, imaging as well as the realization of advanced modulation formats for digital communications, require the generation, analysis and processing of analogue RF signals where both the amplitude and phase of the signals are important. In-phase and quadrature-phase components of a signal can be obtained via a photonic based Hilbert transform [21]. Figure 1 shows a schematic of our ring resonator, with a Q factor of 1.2 million and FSR of $200 \mathrm{GHz}$ [11]. The comb spectrum has a spacing corresponding to the FSR of the microring, had reasonably high coherence, not operating in the chaotic regime [22]. For the microwave demonstrated here this was more than adequate. Figure 1 shows the ring resonator device and the output micro-comb. Figure 2 shows the experimental setup and Figure 3 shows the input and output to a device configured to perform high order RF differentiation. We review a wide range of other novel RF functions based on microcombs [23 - 26]. These devices have significant potential for applications in telecommunications and on-chip WDM optical interconnects for computing and many other applications.
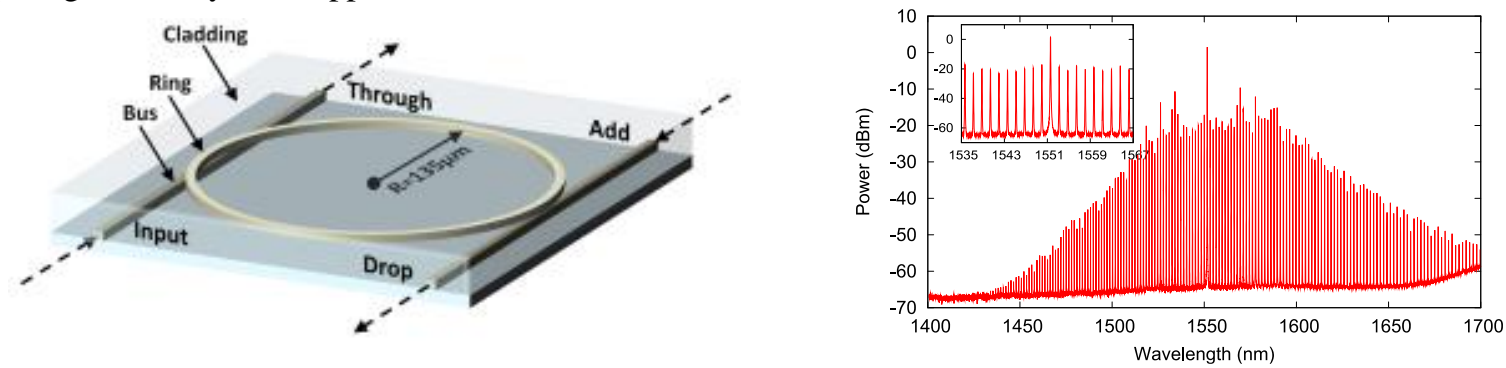

Fig. 1. Integrated ring resonator (top left), output comb spectrum (top right). 


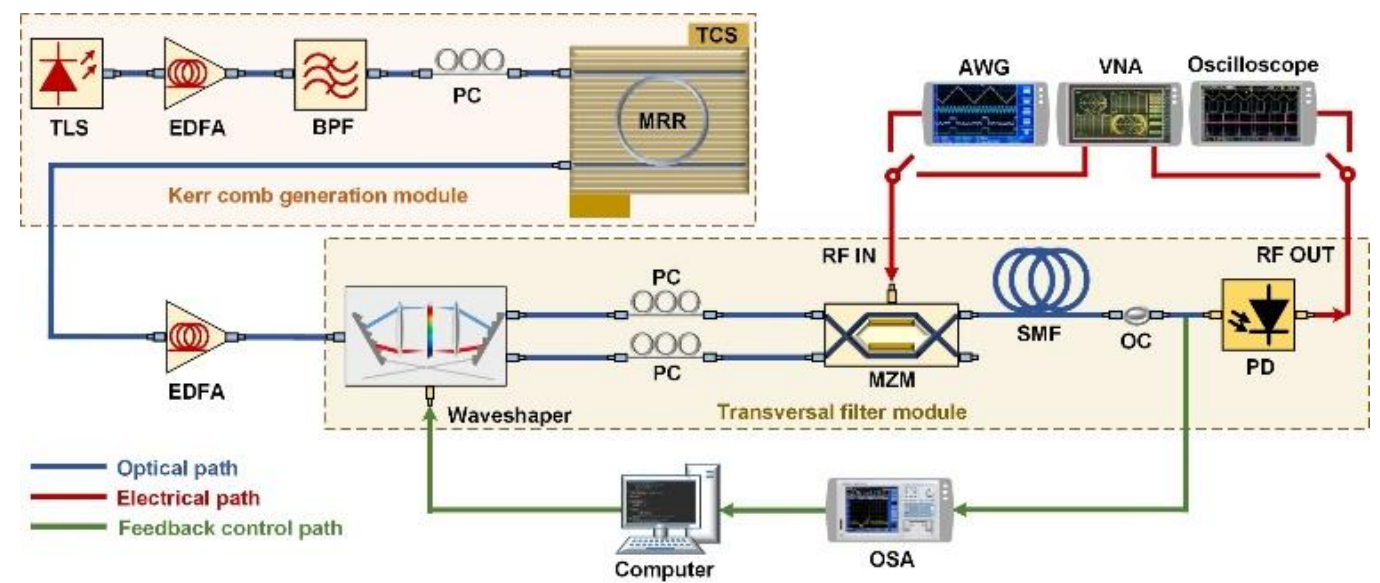

Fig. 2. Experimental setup. TLS = tunable laser source, BPF=bandpass filter, VNA=vector network analyzer, TCS=thermally controlled stage, MZM=machzenhder modulator.

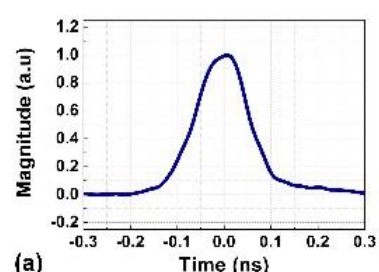

(a)
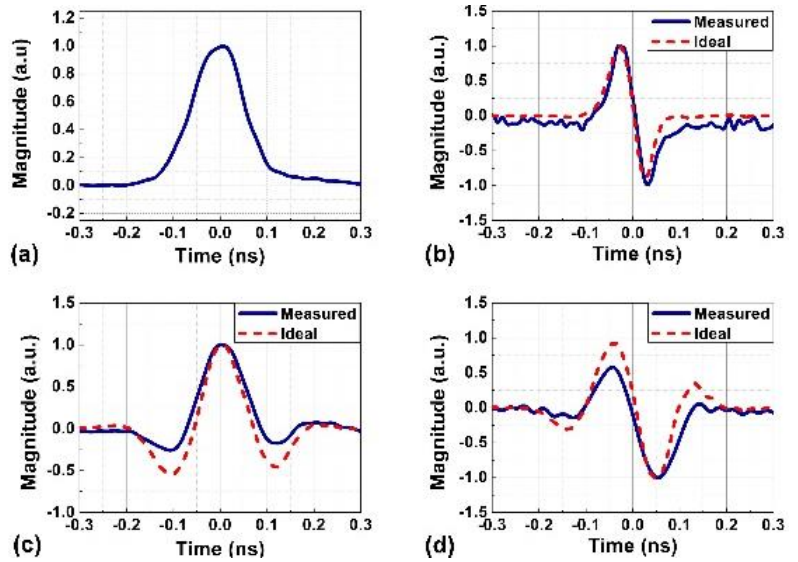

Fig. 3. Experimental measurements of high order differentiation of a microwave pulse a) input pulse b) $1^{\text {st }}$ order derivative, c) second order derivative and d) $3^{\text {rd }}$ order derivative.

[1] Li, F. et al., Optics Express 19, 20364-20371 (2011). DOI: 10.1364/OE.19.020364.

[2] Li, F. et al., Optics Express 18, 3905-3910 (2010). DOI: 10.1364/OE.18.003905.

[3] H.Ji, et.al., Photonics Technology Letters $\underline{22} 1762$ (2010).

[4] Monat, C. et al., Optics express 18 (7), 6831-6840 (2010). DOI: 10.1364/OE.18.006831.

[5] Corcoran, B., et. al., Optics Express 18, (8) 7770-7781 (2010). DOI: 10.1364/OE.18.007770.

[6] V.G. Ta'eed, et al., Optics Letters $\underline{\mathbf{3 0}} 2900$ (2005). DOI: 10.1364/OL.30.002900.

[7] Moss, D.J., et al., "Electronics Letters $\underline{41} 320$ (2005). DOI: DOI: 10.1049/el:20058051.

[8] M. Lamont, et al., Photonics Technology Letters $\underline{\mathbf{1 8}} 1185$ (2006). DOI:10.1109/LPT.2006.874718.

[9] A.Tuniz, G.Brawley, D.J.Moss, B.J.Eggleton,, Optics Express 16 18524 (2008). DOI: 10.1364/OE.16.018524.

[10] Ferrera, M., et al., Nature Photonics 2737 (2008). doi:10.1038/nphoton.2008.228.

[11] L. Razzari et al., Nature Photonics 441 (2010). doi:10.1038/nphoton.2009.236.

[12] M.Peccianti, et al., Nature Communications $\underline{\mathbf{3}} 765$ (2012). doi:10.1038/ncomms1762

[13] A. Pasquazi; et al., Optics Express 20 (24) 27355 - 27362 (2012). DOI: 10.1364/OE.20.027355.

[14] A.Pasquazi; et al., Optics Express 21 (11) 13333-13341 (2013). DOI: 10.1364/OE.21.013333.

[15] A.Pasquazi, et al., Optics Express 18, (8) 7634-7641 (2010). DOI: 10.1364/OE.18.007634.

[16] D. J. Moss, et al., Nature Photonics 7 (8) 597-607 (2013). doi:10.1038/nphoton.2013.183.

[17] M.Peccianti, et al., Optics Express $\underline{\mathbf{1 8}}$, (8) 7625-7633 (2010). DOI: 10.1364/OE.18.007625.

[18] D.Duchesne, et al., Optics Express 18, 923-930 (2010). DOI: 10.1364/OE.18.000923.

[19] Levy, J. S., et al., Nature Photonics $\underline{4}, 37-40$ (2010).

[20] V. R. Supradeepa, et al., Nat. Photonics $\underline{6}$ (3), 186-194 (2012).

[21] T.G .Nguyen, et al., Optics Express 23 (17) 22087 (2015). DOI:10.1364/OE.23.022087

[22] Ferdous, F., et al., Nature Photonics $\mathbf{5}$ 770-776 (2011).

[23] J.Wu et al., IEEE J. of Selected Topics in Quantum Electronics JULY / AUGUST 24 (4) (2018). DOI 10.1109/JSTQE.2018.2805814

[24] X.Xu, et al., Photonics Research Journal $\underline{6}$ (5) May (2018). doi.org/10.1364/PR.99.099999

[25] A.Pasquazi et al., Physics Reports $\underline{729}$ 1-81 January 27 (2018). DOI:10.1016/j.physrep.2017.08.004.

[26] X.Xu, et. al., Optics Express 26, (3) 2569-2583 (2018). 